\title{
Forward and Central Preshower Detectors for the Do Upgrade
}

\author{
Ken Del Signore for the D0 collaboration
}

\author{
University of Michigan, Dept. of Physics, Ann Arbor, Michigan 48109
}

\begin{abstract}
Within the upgraded Do detector at Fermi National Accelerator Laboratory, forward and central preshower detectors will be used for fast level 1 triggering of electrons. These detectors consist of approximately 25000 channels of extruded scintillator strips with embedded wave length shifter fiber readout. Readout is via clear fiber lightguide to Visible Light Photon Counters. An overview of each system will be presented. Results of prototype detectors to cosmic rays will be presented. Scintillator/fiber manufacture and assembly will be discussed.
\end{abstract}

\section{INTRODUCTION}

The upgraded D0 detector [1] will be used in the high luminosity RunII and Tev33 eras at Fermi National Accelerator Laboratory, tentatively scheduled for 2000-2000, 2003-2005, respectively. The D0 Central (CPS) and Forward (FPS) Preshower Detectors [2,3] are designed for use in the Level 1 trigger to tag events which contain high $p_{T}$ electrons. The detectors will also be used offline for tagging soft electrons from $b$ jet decays and as the first layer of the calorimeter to help restore electromagnetic energy resolution otherwise degraded by the solenoid.

The CPS and FPS detectors are made from fine grained scintillator strips, of triangular cross section, with wavelength shifter fiber (WLS) readout. $\mathrm{Pb}$ radiators are used to enhance electron showering. Light collected in the WLS fibers is piped via clear fiber light guides $(\sim 10 \mathrm{~m})$ to the D0 Visible Light Photon Counter (VLPC) system. The VLPC system provides $\sim 80 \% \mathrm{QE}$ and a gain of about $10^{4}$.

Successive strips are rotated 180 degrees such that the triangular cross sections nest into one another. This provides a vernier effect to electron showers and also minimum ionizing particles (mip). A prototype detector, including ten meter clear light guides and readout by HISTE-IV (60\% QE) VLPCs, was installed in the D0 fiber tracker cosmic ray test stand [4]. The light yield was measured to be $\sim 4.5$ photons per millimeter of scintillator traversed. The position resolution to mip tracks using two adjacent strips in a vernier mode was $580 u m$.

\section{DETECTOR GEOMETRY}

The layout of the CPS and FPS detectors can be seen in Figures 1, 3.

The CPS consists of three concentric cylinders, of diameter $28.5^{\prime \prime}$ and length $103^{\prime \prime}$,situated between the new D0 solenoid and the calorimeter. Each cylinder consists of a nested layer of triangular strips sandwiched between 1/32" stainless steel skins. The detector covers the region $|\eta| \leq 1.2$. The strips within each cylinder are situated in axial and $u-v$ stereo views, the stereo angle is $\sim 22.5$ degrees.

CP450, SciFi97: Workshop on Scintillating Fiber Detectors

edited by A. D. Bross, R. C. Ruchti, and M. R. Wayne

() 1998 The American Institute of Physics 1-56396-792-8/98/\$15.00 

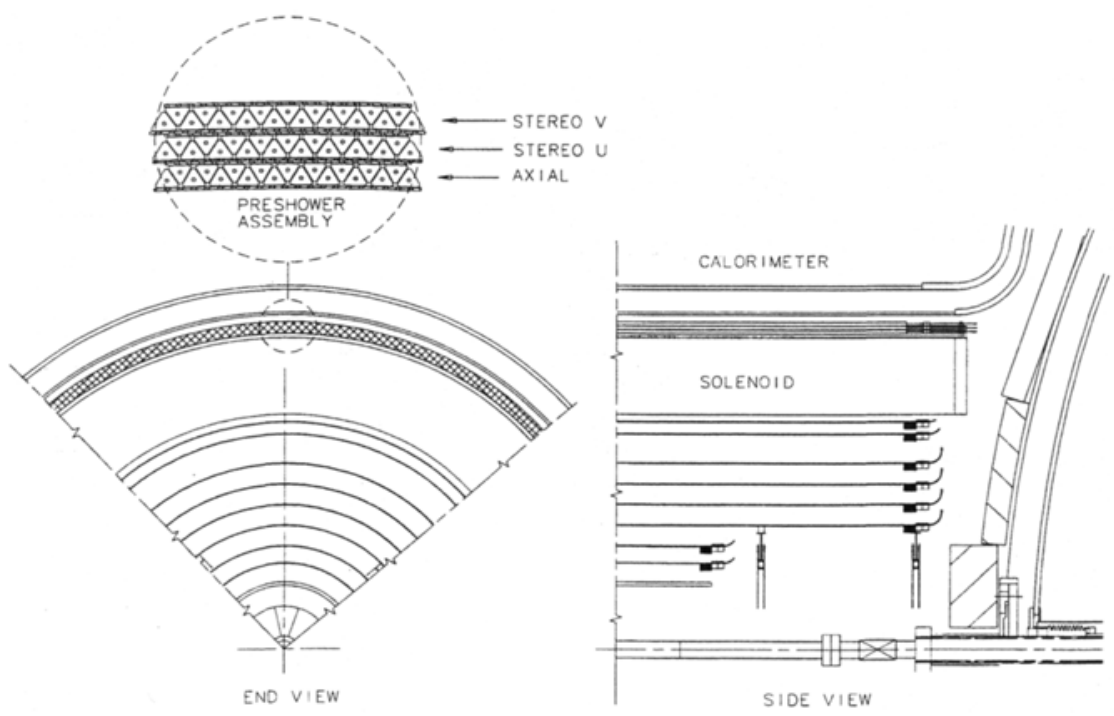

FIGURE 1. End and side views of the Central Preshower Detector. The cylindrical detector is positioned in the $5.5 \mathrm{~cm}$ gap between the solenoid and central calorimeter.

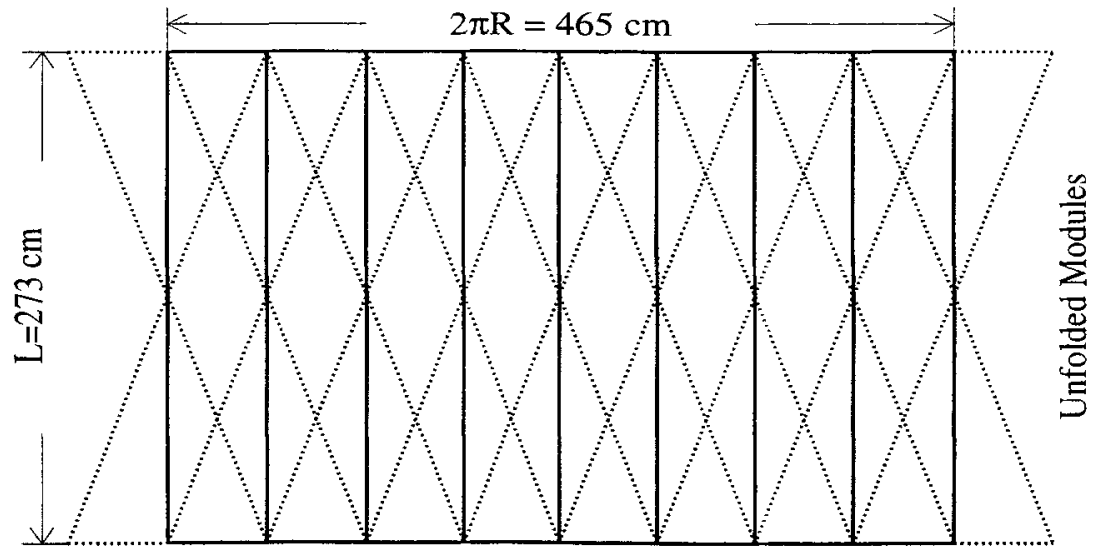

FIGURE 2. The central preshower detector unwrapped in a plane for axial (solid lines) and stereo (dotted lines) octants. Note that each octant crosses three octants in the other two views and that the stereo octant edges are precisely aligned with the axial octant edges. The arrangement yields a stereo angle of $\sim 22.5^{\circ}$. 
A cylindrical $\mathrm{Pb}$ radiator $\left(1 X_{0}\right)$ is mounted between the solenoid and CPS to enhance electron showering. The total radiation length (solenoid $+\mathrm{Pb}$ ) for normally incident electrons is $2 X_{0}$. A Level 1 electron trigger is formed by the spatial match of a hi $p_{T}$ track in the Do Fiber tracker to a shower in an axial strip.

To facilitate detector assembly, each cylindrical layer is formed from eight octant modules. The stereo modules rotate through 90 degrees along the s-axis of the solenoid, so that they attach to every other axial module. Figure 2 shows a rolled out view of the axial and stereo layers.

Each module contains 160 scintillator strips. The WLS fibers within each strip are split in the middle of the detector and readout from each end. The total number of readout channels is $\mathbf{7 6 8 0}$.

The FPS covers the region $1.5 \leq|\eta| \leq 2.5$. In the region $1.65 \leq|\eta| \leq 2.5$ there are four layers in a $u-v$ u-v arrangement, with a $2 X_{0} \mathrm{~Pb}$ absorber between the second and third layers. In the region $1.5 \leq|\eta| \leq 1.65$ only the third and fourth layers are present. The stereo angle is 22.5 degrees. The detector is spherical to conform to the shape of the D0 Endcap Calorimeters.

The $2 \pi$ spherical surface is made from sixteen $\phi$ wedges, each subtending 22.5 degrees, Fig. 4. One WLS fiber reads out each strip. The total channel count is 14,368. The choice of sixteen $\phi$ wedges is a compromise between the total channel count versus decreasing the occupancy of each strip.

A Level 1 electron trigger is formed by requiring a mip sigmal in the front two layers be spatially matched to a shower signal in the back layers. Photons are identified by a shower in the back layers with no signal in the front. Pions will typically leave mip signals in the front and rear.

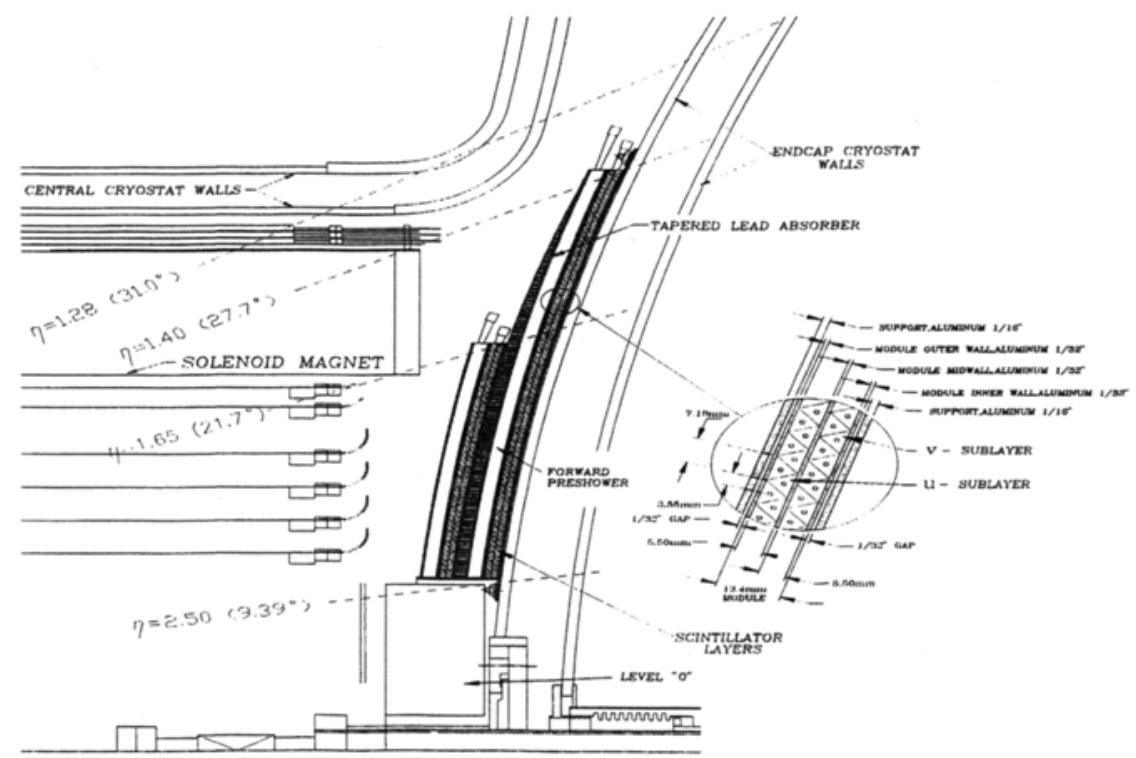

FIGURE 3. Detail of the Forward Preshower Detector. The four layer detector is spherical to match the DO endcap calorimeter.

\section{SCINTILLATOR STRIP MANUFACTURE AND PREPARATION}

The scintillator strips used in the CPS and FPS detectors were manufactured by an extrusion process pioneered by FNAL and DO collaborators. Pellets of polystyrene are impregnated with the primary and secondary fluors. The concentrations used were $1 \%$ PT and $0.015 \%$ DPS. These pellets are then extruded into strips at RDN Corporation [5]. Relatively precise, $\sim 25 \mu \mathrm{m}$, 


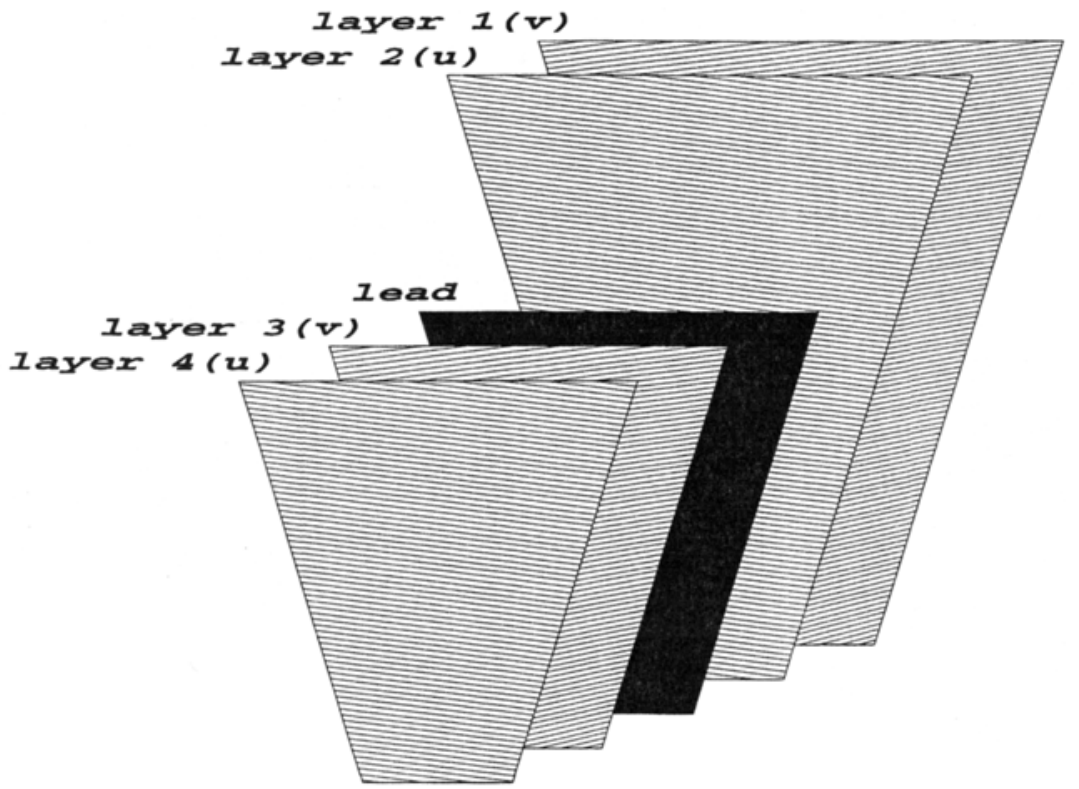

FIGURE 4. Schematic picture of one azimuthal wedge. The lines represent the scintillator strips

tolerances on the strips can be held. A small obstruction in the extrusion flow produces the hole running the length of the strip. Figure 5 shows the strip cross section used for the CPS. The strips were then machine wrapped with aluminized mylar at FNAL.

Following wrapping, the strips are heat formed to the desired shape. Due to both the extrusion and wrapping procedures, the strips tend to be bowed. The heat forming has the additional benefit of relieving stresses within the plastic. The CPS and FPS detectors require unique strip shapes. The CPS axial layer required only straight strips, while the stereo layers require four unique helical shapes. The FPS requires two unique spherical shapes.

The heat forming is accomplished by constraining the strips to the desired shape with appropriate fixtures, then slowly heating to a temperature of $\sim 180-185$ degrees, followed by a slow cool down. A slow heating cycle allows for uniform temperature to be achieved throughout the strip. Care must be taken with the fixture that is holding the strips to the desired shape as any defeets will be impregnated in the strips. Expansion of the plastic is also of concern.

\section{MODULE ASSEMBLY}

The CPS modules were built on three special assembly tables. Each table has a cylindrical surface of appropriate radius and arclength. Modules are built by first epoxing (3M DP-190) a bottom layer of strips onto a stainless steel skin. A polystyrene jig, with registration grooves 
milled in, is set over the strips and registered to precise pins situated on the table surface. This assembly is then vacuum bagged. The uniform pressure applied by the vacuum bag seats each strip into the jig. The strip to strip registration achieved in this manor is $\sim 50 \mu \mathrm{m}$. After an overnight cure, a top layer of strips is set in place using the bottom layer for registration.

The mylar wrapping at the ends of the strips tended to be non-uniform. This necessitated gluing the strips on with $\sim 4$ " of excess length. The strips were cut to the proper length with a diamond disk in a dremel.

Following this, each hole was cleaned and inspected for obstructions. Fiber bundles, in groups of sixteen, were then inserted. Care had to be taken during insertion as the fibers were prone to damage. After insertion a visual inspection of the polished connector, for non-uniform brightness of individual fibers, proved to be a robust test for damaged fibers.

The FPS modules are constructed in a slightly different manor, owing to the differences in geometry [3].

A spherical assembly table is made on a large lathe. Onto the surface are set jigs that constrain the edges of the first and last strips and the ends of every fifth strip of the bottom layer. The top and bottom layer are glued at same time between two .01 " g-10 substrates. A vacuum bag compresses the assembly during curing. With the first, last, and every fifth strip constrained to the proper position, and given the natural symmetry of the top and bottom layers, the strips align themselves during compression. Prototypes built in this manor have a strip to strip registration of $.25 \mu \mathrm{m}$.

The final trapezoidal shape is then cutout using a CNC water jet cutter. This technique employs a thin jet of water deployed from a small nozzle at $\sim 50,000$ psi. Outer dimensions can

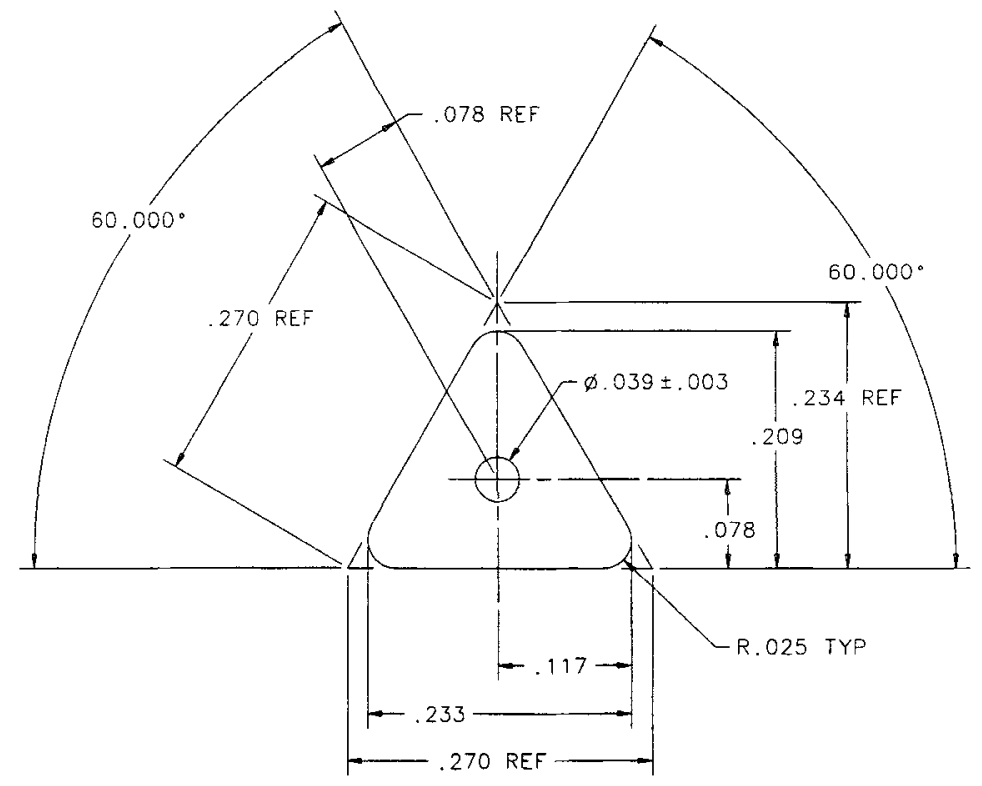

FIGURE 5. Cross sectional dimensions of the equilateral triangular strip with a hole in the center for housing a WLS fiber 
be held to $\sim .015 "$. This procedure sometimes leaves a small amount of sand (added to the water as an abrasive) in the WLS fiber holes, which necessitates cleaning by an air gun.

Full details of CPS and FPS detector assembly, mounting hardware, etc, can be found in $[2,3]$.

\section{FIBER BUNDLE/CONNECTOR PREPARATION}

The WLS fibers used for both the FPS and CPS are Kurrary Y-11, 250ppm, S-type [6]. The non readout end of the fibers are ice-polished in batches on approximately 400 . The polished end is then silvered by $\mathrm{Al}$ sputtering. A small bead of Elmers glue was applied to the silvering as a protectant. Care had to be taken with the fibers as the silvering was still somewhat fragile. Approximately $70 \%$ of the light incident on the silvering is reflected back into the fiber [4].

The connectors used to transition the WLS fiber to clear fiber were developed by D0 collaborators at the University of Illinois at Chicago (UIC) [7]. The connectors are made by an injection molding process that allows accurate reproduction in large quantities. Figure 6 shows a schematic of the FPS 16 channel connector. The connectors, made of black ABS plastic, can be made to tolerances of $\sim .001 "$, and offer typical light transmission of $95 \% \pm 1 \%$.
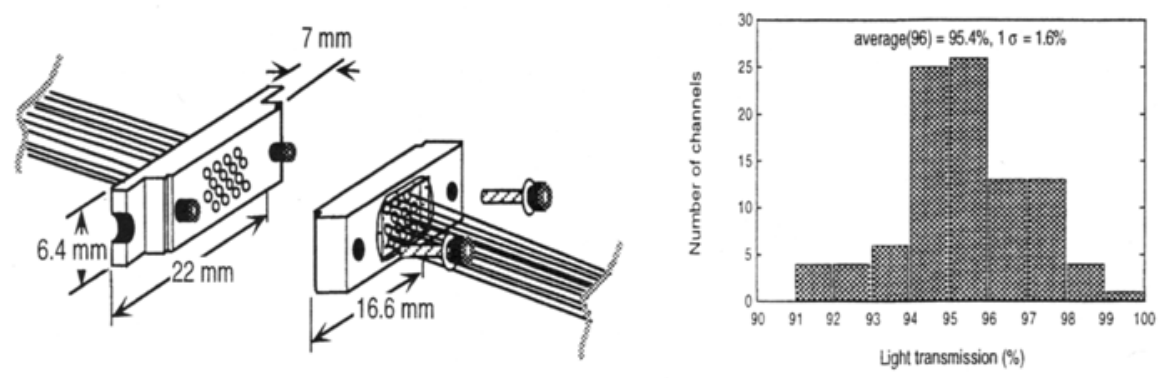

FIGURE 6. Schematic of FPS 16 channel WLS-clear fiber connector and a typical measurement of transmission efficiency.

\section{REFERENCES}

1. The Do Upgrade, FERMILAB-Pub-96/357-E.

2. Design Report of the Central Preshower Detector for the Do Upgrade, D0-Note 3014.

3. Technical Design Report of the Forward Preshower Detector for the Do Upgrade, D0-Note 3445.

4. M. Adams, et al., A New Detector Technique Using Triangular Scintillating Strips to Achieve Precise Position Measurements for Minimum Ionizing Particles, FERMILAB-Pub-95/285-E, September 1995, to be submitted to Nucl. Instrum, and Meth. A.

5. RDN Corporation, 160 Covington Drive, Bloomingdale, IL 60108, (630) 595-4876

6. Kurraray Intl., 200 Park Ave., New York, NY 10166 (212)986-2230

7. S. Margulies and M. Chung, Development of a multichannel fiber-to-fiber connector for the Do upgrade tracker, Photoelectronic Detectors, Cameras, and Systems, eds. C.B. Johnson and E.J. Fenyves, Proc. SPIE 2551, pp. 10-16, 1995. M. Chung and S. Margulies, Development of a multichannel fiber-to-fiber connector for the DO upgrade tracker, 1995 IEEE Nuclear Science Symposium, Vol 43, No. 3, June 1996 